\title{
Experimental Simulation of Spin Squeezing by Nuclear Magnetic Resonance
}

\author{
Suddhasattwa Sinha, Joseph Emerson, Nicolas Boulant, \\ Evan M. Fortunato, Timothy F. Havel, ${ }^{1}$ and David G. Cory
}

Received May 3, 2004; accepted July 14, 2004

We report on the experimental simulation of spin squeezing using a liquid-state nuclear magnetic resonance (NMR) quantum information processor. This was done by identifying the energy levels within the symmetric subspace of a system of $n$ spin-1/2 nuclei with the energy levels of the simulated spin-(n/2) system. The results obtained for our simulations of spin-1 and spin-3/2 systems are consistent with earlier theoretical studies of spin squeezing, and illustrate interesting relations between the degree of squeezing and the strength of the correlations among the underlying spin-1/2 particles.

KEY WORDS: Coherent and squeezed spin states; nuclear magnetic resonance; quantum information processing.

PACS: 03.65.Bz; 03.67.-a; 03.67.Lx.

\section{INTRODUCTION}

The minimum uncertainty associated with complementary observables is given by the uncertainty relations. For example, the position and momentum fluctuations in a coherent state of the quantum harmonic oscillator are both equal to the quantum limit $\hbar / 2$. States for which the fluctuations in one of these observables is less than the standard quantum limit of $\hbar / 2$, while the fluctuations in the complementary observable increase so as to satisfy the uncertainty relation, are called "squeezed states". ${ }^{(1,2)}$ Thus a squeezed state can be visualized as an ellipse of constant uncertainty in phase space.

Squeezed spin states have been defined using analogous criteria, ${ }^{(3-9)}$ and several experimental demonstrations of spin squeezing have been published. They include interaction of collection of atoms with squeezed

\footnotetext{
${ }^{1}$ To whom correspondence should be addressed. E-mail: tfhavel@mit.edu
} 
radiation, ${ }^{(10)}$ the displacement of two optical lattices with respect to each other, ${ }^{(11)}$ and collisional interactions between the atoms in a Bose-Einstein condensate. ${ }^{(3,5,12)}$ Spin squeezing by quantum non-demolition measurements has also been proposed. ${ }^{(13)}$

A spin- $1 / 2$ is always in a coherent state, but it is possible to squeeze the "effective" higher spin with $j=n / 2$ that lives within the symmetric manifold of states in a system of $n>1$ spins each with $j=1 / 2 .^{(14,15)}$ In this paper we describe experimental realizations of squeezed states of these simulated higher order spins on a liquid-state nuclear magnetic resonance (NMR) quantum information processor, using the method suggested by Kitagawa. ${ }^{(4)}$ First, we review the properties of coherent spin states and Kitagawa's method for creating squeezed spin states, along with the representation of a spin- $j$ system for $j=1$ and $3 / 2$ within the totally symmetric subspace of the Hilbert space of $2 j$ spin- $1 / 2$ particles. We then describe the NMR implementation of the method and the measures used to access its overall precision, after which the experimental results of squeezing are presented and the level of control attained is discussed. We end by verifying the relation between the degree of squeezing of the simulated spin- $j$ system and the degree of pure state entanglement among the underlying spin-1/2 particles, as quantified by various well-established entanglement measures. ${ }^{(5,11,16)}$

\section{SIMULATION OF SPIN SQUEEZING IN A MULTI-SPIN-1/2 SYSTEM}

Throughout the remainder of this paper we will work with units such that $\hbar=1$. Coherent spin states (CSS) may then be defined by the following properties: (i) The uncertainty relation for the total angular momentum operator $J$ becomes saturated, i.e., $\Delta J_{x} \Delta J_{y}=(1 / 2)\left|\left\langle J_{z}\right\rangle\right|$, where $(x, y, z)$ label the coordinate axes. (ii) The absolute expectation value of the spin in the direction of polarization, e.g. $\left|\left\langle J_{z}\right\rangle\right|$, is maximum and equal to $j$. (iii) The spin uncertainties are equally distributed in any two orthogonal directions in the plane normal to the direction of polarization, e.g., $\Delta J_{x}=\Delta J_{y}$. Kitagawa and $\mathrm{Ueda}^{(4)}$ proposed that a spin state is squeezed if the minimum spin uncertainty in the $(x, y)$-plane is less than the standard quantum limit of $\sqrt{j / 2}$. Since a squeezed spin state (SSS) is not related to a CSS by a simple rotation, the polarization of an SSS is less than maximum, e.g., $\left|\left\langle J_{z}\right\rangle\right|<j$.

To create a SSS, a "non-linear" operation must be applied, i.e. one that involves products or powers of the spin operators $J_{x}, J_{y}$ and $J_{z}$ in its Hamiltonian $H$. Kitagawa and Ueda proposed two methods for squeezing 


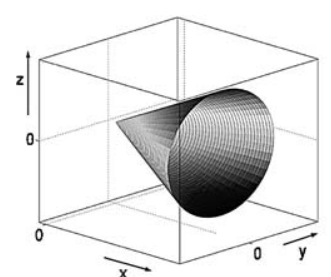

(i)

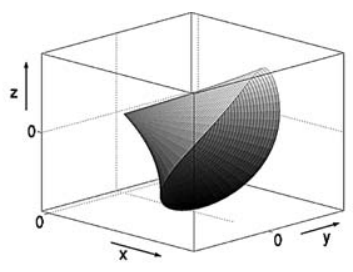

(ii)

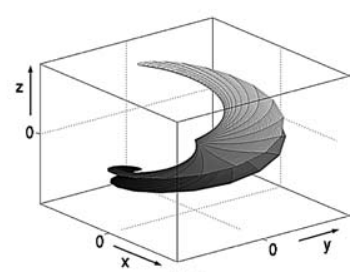

(iii)

Fig. 1. (i) A coherent state spin- $j$ vector with expectation values $\left[\left\langle J_{x}\right\rangle,\left\langle J_{y}\right\rangle,\left\langle J_{z}\right\rangle\right]=[1,0,0]$ may be visualized as a coherent superposition of angular momentum vectors on a cone about the $x$-axis, all with a projection of $j / 2$ along that axis. (ii) After squeezing via the one-axis mechanism (see text), the cone representing the state of a spin-1 particle is elliptical, with its squeezed (minor) axis making an angle of $\pm \pi / 4$ with the $y, z$-axes. (iii) In the maximally squeezed state of a spin-1 particle the cone has been folded into a nearly degenerate ruled surface.

a CSS: (i) $H=J_{z}^{2}$ (the one-axis twisting mechanism), or (ii) $H=J_{x} J_{y}+$ $J_{y} J_{x}$ (the two-axis twisting mechanism). The second Hamiltonian can be applied directly to any CSS to squeeze it, whereas the first requires that the CSS be rotated to the $(x, y)$-plane before the propagator $\exp \left(\mathrm{i} k J_{z}^{2}\right)$ is used to squeeze it, where $k$ is real number which we will call the squeezing parameter. Figure 1 illustrates the one-axis process for $j=1$ polarized along $\mathrm{x}$, in which case maximum squeezing in the $(y, z)$-plane is obtained when $k=\pi / 2$. This one-axis method was used in the NMR experiments described below.

A system of two spin-1/2 particles has four basis states. In the coupled representation (in Table 1), the three symmetric states span a subspace that transforms under identical rotations of both spins like a single spin-1. Similarly, a system of three spin-1/2 particles has eight basis states, which span a symmetric subspace that transforms like a single spin$3 / 2$, as well as two spin- $1 / 2$ subspaces with lower symmetry. The four

Table 1. The Representation of a Spin-1 by Two Spin-1/2 Particles

Uncoupled representation $\left(\left|m^{1} m^{2}\right\rangle\right) \quad$ Coupled representation $(|j, m\rangle)$
$|00\rangle=|\uparrow \uparrow\rangle$
$|1,1\rangle=|\uparrow \uparrow\rangle$
$|01\rangle=|\uparrow \downarrow\rangle$
$|1,0\rangle=\frac{1}{\sqrt{2}}[|\uparrow \downarrow\rangle+|\downarrow \uparrow\rangle]$
$|10\rangle=|\downarrow \uparrow\rangle$
$|1,-1\rangle=|\downarrow \downarrow\rangle$
$|11\rangle=|\downarrow \downarrow\rangle$
$|0,0\rangle=\frac{1}{\sqrt{2}}[|\uparrow \downarrow\rangle-|\downarrow \uparrow\rangle]$ 
Table 2. Coupled Representation $(|j, m\rangle)$ of the Basis States Spanned by Three Spin-1/2 Particles

Spin-3/2 subspace basis

Spin-1/2 subspace basis

\begin{tabular}{cc}
\hline$\left|\frac{3}{2}, \frac{3}{2}\right\rangle=|\uparrow \uparrow \uparrow\rangle$ & $\left|\frac{1}{2}, \frac{1}{2}\right\rangle=\frac{1}{\sqrt{6}}[2|\uparrow \uparrow \downarrow\rangle-|\uparrow \downarrow \uparrow\rangle-|\downarrow \uparrow \uparrow\rangle]$ \\
$\left|\frac{3}{2}, \frac{1}{2}\right\rangle=\frac{1}{\sqrt{3}}[|\uparrow \uparrow \downarrow\rangle+|\uparrow \downarrow \uparrow\rangle+|\downarrow \uparrow \uparrow\rangle]$ & $\left|\frac{1}{2},-\frac{1}{2}\right\rangle=\frac{1}{\sqrt{6}}[|\uparrow \downarrow \downarrow\rangle+|\downarrow \uparrow \downarrow\rangle-2|\downarrow \downarrow \uparrow\rangle]$ \\
$\left|\frac{3}{2},-\frac{1}{2}\right\rangle=\frac{1}{\sqrt{3}}[|\downarrow \downarrow \uparrow\rangle+|\uparrow \downarrow \downarrow\rangle+|\downarrow \uparrow \downarrow\rangle]$ & $\left|\frac{1}{2}, \frac{1}{2}\right\rangle=\frac{1}{\sqrt{2}}[|\uparrow \downarrow \uparrow\rangle-|\downarrow \uparrow \uparrow\rangle]$ \\
$\left|\frac{3}{2},-\frac{3}{2}\right\rangle=|\downarrow \downarrow \downarrow\rangle$ & $\left|\frac{1}{2},-\frac{1}{2}\right\rangle=\frac{1}{\sqrt{2}}[|\uparrow \downarrow \downarrow\rangle-|\downarrow \uparrow \downarrow\rangle]$ \\
\hline
\end{tabular}

states listed on the left-hand side column of Table 2 are the coupled representation of the spin-3/2 subspace.

From the uncoupled and coupled representations, we see that there is a linear mapping from the spin- $j$ subspaces into the combined $2 j$ spin- $1 / 2$ systems. These mappings induce the mappings between the spin- $j$ operators and products of spin-1/2 operators (denoted here by $I_{\text {axis }}^{\text {spin }}$ ), which for $j=1$ is simply

$$
J_{x}=I_{x}^{1}+I_{x}^{2}, \quad J_{y}=I_{y}^{1}+I_{y}^{2}, \quad J_{z}=I_{z}^{1}+I_{z}^{2} .
$$

For $j=3 / 2$, on the other hand, we obtain

$$
\begin{aligned}
& J_{x}=I_{x}^{1} I_{x}^{2} I_{x}^{3}\left(2+\frac{2}{3}\left(I_{x}^{1} I_{x}^{2}+I_{x}^{1} I_{x}^{3}+I_{x}^{2} I_{x}^{3}\right)-\frac{8}{3}\left(I^{1} \cdot I^{2}+I^{1} \cdot I^{3}+I^{2} \cdot I^{3}\right)\right), \\
& J_{y}=I_{y}^{1} I_{y}^{2} I_{y}^{3}\left(2+\frac{2}{3}\left(I_{y}^{1} I_{y}^{2}+I_{y}^{1} I_{y}^{3}+I_{y}^{2} I_{y}^{3}\right)-\frac{8}{3}\left(I^{1} \cdot I^{2}+I^{1} \cdot I^{3}+I^{2} \cdot I^{3}\right)\right), \\
& J_{z}=I_{z}^{1} I_{z}^{2} I_{z}^{3}\left(2+\frac{2}{3}\left(I_{z}^{1} I_{z}^{2}+I_{z}^{1} I_{z}^{3}+I_{z}^{2} I_{z}^{3}\right)-\frac{8}{3}\left(I^{1} \cdot I^{2}+I^{1} \cdot I^{3}+I^{2} \cdot I^{3}\right)\right) .
\end{aligned}
$$

Given the apparent complexity of implementing these operations, it was decided instead to implement the far simpler analoges of Eq. (1), namely:

$$
\tilde{J}_{x}=I_{x}^{1}+I_{x}^{2}+I_{x}^{3}, \quad \tilde{J}_{y}=I_{y}^{1}+I_{y}^{2}+I_{y}^{3}, \quad \tilde{J}_{z}=I_{z}^{1}+I_{z}^{2}+I_{z}^{3} .
$$

It is easily shown that $J_{x}, J_{y}$, and $J_{z}$ are contained within the algebra generated by $\tilde{J}_{x}, \tilde{J}_{y}$, and $\tilde{J}_{z}$, and that the latter satisfy the usual angular momentum commutation relations. In fact, since they have two extra pairs of identical eigenvalues $\pm 1 / 2$, they are the sum of the angular momentum operators for a spin-3/2 mixed with those for a pair of spin-1/2. As a result, it was not possible to squeeze the corresponding coherent states to the amount that would have been possible if a pure spin- $3 / 2$ representation had been used. 
It is apparent that for $j=1, \exp \left(-\mathrm{i} k J_{z}^{2}\right)$ is an evolution $\exp \left(-\mathrm{i} k 4 I_{z}^{1} I_{z}^{2}\right)$ under the bilinear Hamiltonian associated with the scalar coupling interaction between spins in NMR, up to an overall phase factor. Thus the traceless part of the pseudopure state as a function of the squeezing parameter, and its spin-1 pure state equivalent, are

$$
\begin{aligned}
e^{-\mathrm{i} k 4 I_{z}^{1} I_{z}^{2}}\left(\frac{1}{2} I_{x}^{1}+\frac{1}{2} I_{x}^{2}+I_{x}^{1} I_{x}^{2}\right) e^{\mathrm{i} k 4 I_{z}^{1} I_{z}^{2}}= & \cos (k)\left(\frac{1}{2} I_{x}^{1}+\frac{1}{2} I_{x}^{2}+I_{x}^{1} I_{x}^{2}\right) \\
& -\sin (k)\left(I_{y}^{1} I_{z}^{2}+I_{z}^{1} I_{y}^{2}\right) \\
\Leftrightarrow e^{-\mathrm{i} k J_{z}^{2}} \frac{1}{2}\left(J_{x}+J_{x}^{2}\right) e^{\mathrm{i} k J_{z}^{2}}= & \cos (k) \frac{1}{2} J_{x}+\frac{1}{2} J_{x}^{2}-\mathrm{i} \sin (k) \frac{1}{2}\left[J_{x}, J_{z}^{2}\right],
\end{aligned}
$$

where [,] is the commutator. For $j=3 / 2$, on the other hand, we find that

$$
\begin{aligned}
J_{z}^{2} & =\frac{5}{8} I_{8}+\frac{1}{6}\left(\boldsymbol{I}^{1} \cdot \boldsymbol{I}^{2}+\boldsymbol{I}^{1} \cdot \boldsymbol{I}^{3}+\boldsymbol{I}^{2} \cdot \boldsymbol{I}^{3}\right)+2\left(I_{z}^{1} I_{z}^{2}+I_{z}^{1} I_{z}^{3}+I_{z}^{2} I_{z}^{3}\right), \\
& =\tilde{J}_{z}^{2}+\frac{1}{6}\left(\boldsymbol{I}^{1} \cdot \boldsymbol{I}^{2}+\boldsymbol{I}^{1} \cdot \boldsymbol{I}^{3}+\boldsymbol{I}^{2} \cdot \boldsymbol{I}^{3}-\frac{3}{4} I_{8}\right) .
\end{aligned}
$$

It follows that $\exp \left(-i k J_{z}^{2}\right)$ is, to a fairly good approximation, the same as three equal scalar coupling evolutions up to phase, and the (pure) state as a function of the squeezing parameter becomes

$$
\begin{aligned}
& e^{-\mathrm{i} k 4\left(I_{z}^{1} I_{z}^{2}+I_{z}^{1} I_{z}^{3}+I_{z}^{2} I_{z}^{3}\right) \frac{1}{2}\left(I_{8}+2 I_{x}^{1}\right) \frac{1}{2}\left(I_{8}+2 I_{x}^{2}\right) \frac{1}{2}\left(I_{8}+2 I_{x}^{3}\right) e^{\mathrm{i} k 4\left(I_{z}^{1} I_{z}^{2}+I_{z}^{1} I_{z}^{3}+I_{z}^{2} I_{z}^{3}\right)}}=\frac{1}{2}\left(I_{8}+I_{x}^{1}\left(I_{8}-4 I_{z}^{2} I_{z}^{3}\right)+\cos (2 k) I_{x}^{1}\left(I_{8}+4 I_{z}^{2} I_{z}^{3}\right)-\sin (2 k) I_{y}^{1}\left(I_{z}^{2}+I_{z}^{3}\right)\right) \cdots \\
& \frac{1}{2}\left(I_{8}+I_{x}^{2}\left(I_{8}-4 I_{z}^{1} I_{z}^{3}\right)+\cos (2 k) I_{x}^{2}\left(I_{8}+4 I_{z}^{1} I_{z}^{3}\right)-\sin (2 k) I_{y}^{2}\left(I_{z}^{1}+I_{z}^{3}\right)\right) \cdots \\
& \frac{1}{2}\left(I_{8}+I_{x}^{3}\left(I_{8}-4 I_{z}^{1} I_{z}^{2}\right)+\cos (2 k) I_{x}^{3}\left(I_{8}+4 I_{z}^{1} I_{z}^{2}\right)-\sin (2 k) I_{y}^{3}\left(I_{z}^{1}+I_{z}^{2}\right)\right) \\
& \leftrightarrow \frac{1}{16}\left(e^{-\mathrm{i} k / 4}\left(18-J_{z}^{2}\right)+e^{-\mathrm{i} k 9 / 4}\left(2-J_{z}^{2}\right)\right) \frac{1}{48}\left(-3 I_{8}-2 J_{x}+12 J_{x}^{2}+8 J_{x}^{3}\right) \cdots \\
& \frac{1}{16}\left(e^{\mathrm{i} k / 4}\left(18-J_{z}^{2}\right)+e^{\mathrm{i} k 9 / 4}\left(2-J_{z}^{2}\right)\right),
\end{aligned}
$$

where in the last line we have expanded both the exponential $\exp \left(-\mathrm{i} k J_{z}^{2}\right)$ and the initial coherent state along $x$ in terms of angular momentum operators. Finally, the CSS $|0\rangle$ is, for both values of $j$, the same as the Zeeman ground state $|\uparrow \uparrow\rangle$ or $|\uparrow \uparrow \uparrow\rangle$ in the uncoupled representation.

\section{THE NMR IMPLEMENTATION OF SQUEEZED SPIN STATES}

The implementation of spin squeezing was carried out on a liquidstate NMR quantum information processor, using the two spin-1/2 hydrogen nuclei of 2,3-dibromo-thiophene (see Fig. 2) to represent the spin-1 system, while the spin-3/2 system was represented using the three 

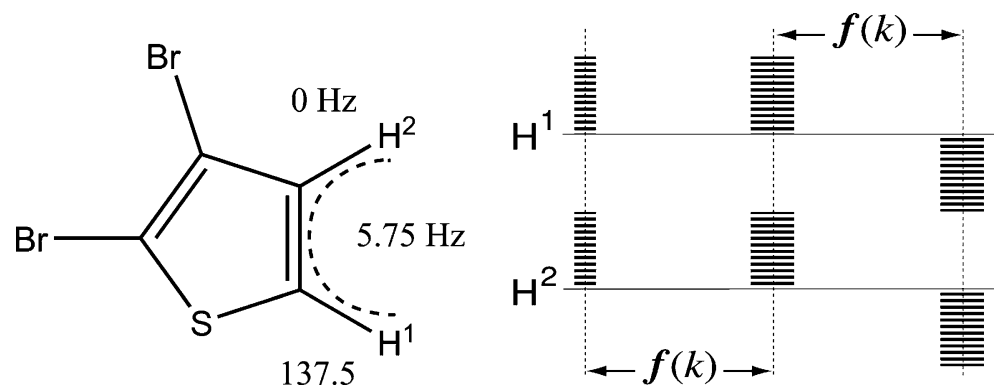

Fig. 2. The molecular structure of 2,3-dibromo-thiophene is shown on the left, with the two hydrogen atoms used for the spin-1 simulations numbered as in the main text. The coupling strength $J$ between them and the chemical shift of the first compared to the second are also shown. The diagram on the right shows the pulse sequence used to implement spin squeezing for a given parameter $k$, where $f(k) \equiv k /(2 \pi J)$, narrow rectangles indicate $\pi / 2$ $y$-rotations, the broad rectangles $y$-rotations by $\pi$, and the rectangles point downwards for pulses effecting rotations by the negatives of these angles.

carbon atoms of a ${ }^{13} \mathrm{C}$-labeled sample of alanine (see Fig. 3). Both the experiments were carried out on a Bruker AVANCE-400 spectrometer in a field of ca. $11.7 \mathrm{~T}$. In the case of the two-spin experiment, frequencyselective pulses were used to rotate single spins, and hard $\pi$-pulses to refocus unwanted chemical shifts. To compensate for pulse imperfections, composite pulses were employed instead of the standard $\pi$-pulse. ${ }^{(17)}$ In the three-spin experiment, strongly modulating pulses were used to more accurately perform the desired unitary operations. ${ }^{(18,19)}$ Unlike low-power "soft" pulses, these pulses average out unwanted evolution, are shorter in time and hence also reduce relaxation effects.

The $I_{z}^{1} I_{z}^{2}$ operator used to squeeze the spin-1 system was implemented using $\pi$-pulses to refocus the Zeeman evolution of the spins while allowing the scalar coupling between them to evolve, in the standard fashion. ${ }^{(20)}$ Because the coupling between spins 1 and 3 of the alanine system is so small $\left(J^{13}=-1.29 \mathrm{~Hz}\right)$, the coupling between them was generated out of the much stronger 1,2 and 2,3 couplings. ${ }^{(21)}$ The sum of the three scalar coupling terms of the form $I_{z}^{\ell} I_{z}^{m}$ was taken as an approximation to $J_{z}^{2}$ in the spin-3/2, as indicated in Eq. (5). As usual in NMR quantum information processing, ${ }^{(22,23)}$ pseudo-pure states were used to represent the dynamics of pure states. These were obtained using spatial averaging techniques based on magnetic field gradients. ${ }^{(24,25)}$

Even though the decoherence times in liquid-state NMR are long, the intrinsic decoherence rates of the spins still impose limits on the accuracy of the experimental results. The $T_{2}$ relaxation rates in 2,3-dibromothiophene were $3.2 \mathrm{~s}^{-1}$ for both of the hydrogen spins, while in alanine 

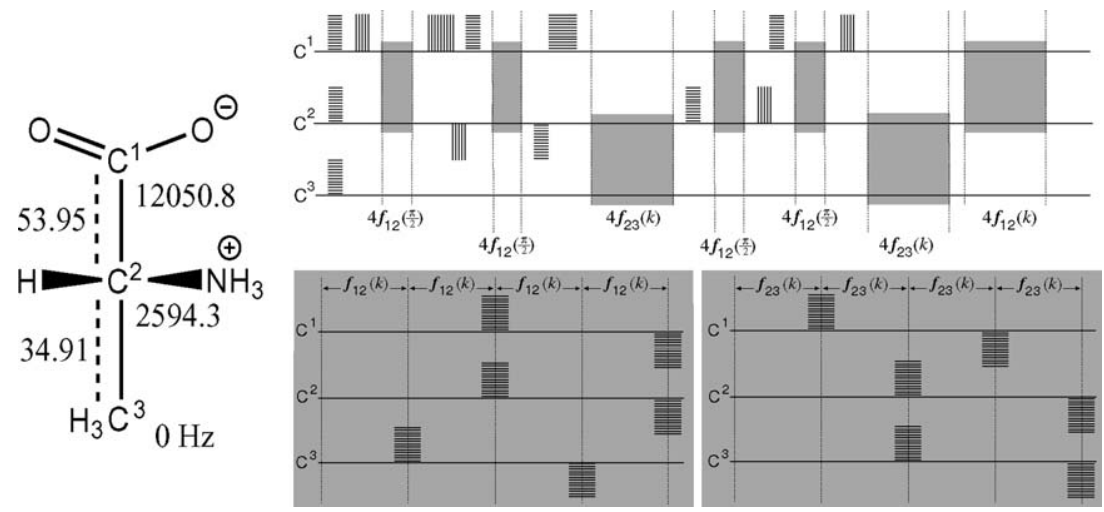

Fig. 3. The molecular structure of alanine is shown on the left, with the three carbon-13 atoms used for the experiments numbered as in the text. The numbers next to the dashed lines are the corresponding coupling constants (in $\mathrm{Hz}$ ), while the numbers next to the carbons are their chemical shifts relative to the third (in $\mathrm{Hz}$ ). On the top right is a schematic diagram of the pulse sequence used, where the horizontal striping indicates an $y$-pulse, vertical striping an $x$-pulse, the narrower pulses induce rotations by $\pi / 2$, and the wider ones by $\pi$ (or the negatives thereof if the pulse is below the spin's line). The grey boxes indicate one of the two "modules" show on the bottom right, which allow a $I_{z}^{m} I_{z}^{n}$ evolution by the angle ( $k$ or $\pi / 2$ ) shown on the bottom of the pulse sequence diagram while refocussing the chemical shifts and the coupling between the pairs not contained in the grey box. The functions which relate the delay time to the squeezing parameter $k$ is given by $f_{m n}(k) \equiv k /\left(4 \pi J^{m n}\right)$ for $[m, n]=[1,2],[2,3]$.

these rates were $0.55,0.42$ and $0.80 \mathrm{~s}^{-1}$ for the $\mathrm{C}^{1}, \mathrm{C}^{2}$ and $\mathrm{C}^{3}$ spins, respectively. The products of the shortest of these decoherence times, multiplied by the weakest coupling constant used, were better than 15 for both 2,3-dibromo-thiophene and alanine, which allows about $30 \mathrm{c}-\mathrm{NOT}$ gates before decoherence begins to seriously degrade the quality of the results. The amount of time needed for the longest experiments reported here was only about one third of this.

The accuracy of the experiments is further affected by systematic errors like imperfect calibration of the pulses, off-resonance effects and RF inhomogeneity. ${ }^{(20)}$ The effects of these errors may be seen in the density matrices of the final states, which in turn were determined by full state tomography. ${ }^{(26)}$ Although plots of these density matrices (see Figs. 4 and 5) provide a visual overview of the results, a more quantitative summary of the overall accuracy may be obtained by calculating the correlation between the theoretically expected and experimentally determined density matrices $\rho_{\text {the }}$ and $\rho_{\text {fin }}$, respectively. This is defined as

$$
C=\frac{\operatorname{tr}\left(\rho_{\mathrm{fin}} \rho_{\mathrm{the}}\right)}{\sqrt{\operatorname{tr}\left(\rho_{\mathrm{fin}} \rho_{\mathrm{fin}}\right) \operatorname{tr}\left(\rho_{\mathrm{the}} \rho_{\mathrm{the}}\right)}} .
$$




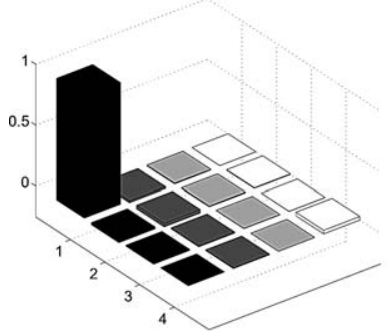

(i)

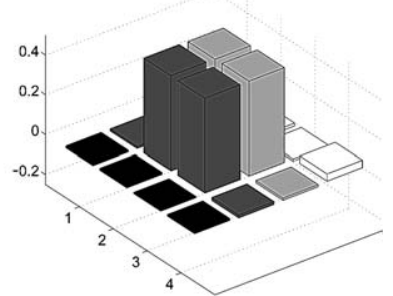

(ii)

Fig. 4. (i) The real part of the experimental density matrix representing the initial pseudopure state $|00\rangle$ for the spin-1 experiments. (ii) The real part of the experimental density matrix representing the maximally squeezed state of the effective spin-1 particle.

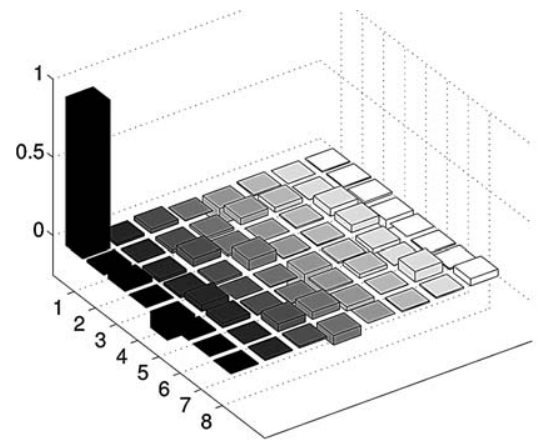

(i)

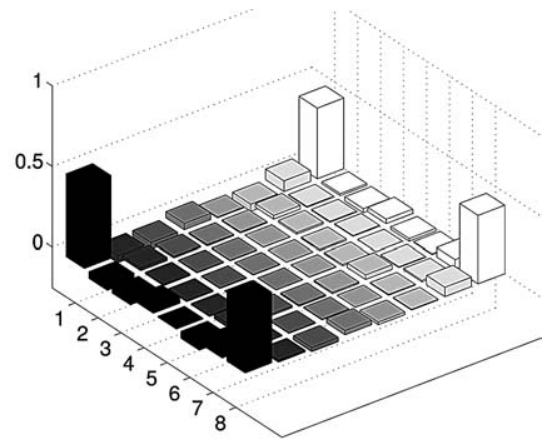

(ii)

Fig. 5. (i) The real part of the experimental density matrix representing the initial pseudopure state $|000\rangle$ for the spin-3/2 experiments. (ii) The real part of the density matrix when the squeezing parameter $k=\pi / 2$, at which point a GHZ state is obtained. ${ }^{(27)}$

To also include an estimate of the precision of the experiment, the amount of signal (or polarization) lost during the experiment must also be taken into account. This leads to a metric called the attenuated correlation, ${ }^{(18)}$ namely

$$
C_{\text {att }}=C \frac{\sqrt{\operatorname{tr}\left(\rho_{\text {fin }} \rho_{\text {fin }}\right)}}{\sqrt{\operatorname{tr}\left(\rho_{\text {ini }} \rho_{\text {ini }}\right)}}=\frac{\operatorname{tr}\left(\rho_{\text {fin }} \rho_{\text {the }}\right)}{\sqrt{\operatorname{tr}\left(\rho_{\text {ini }} \rho_{\text {ini }}\right) \operatorname{tr}\left(\rho_{\text {the }} \rho_{\text {the }}\right)}} .
$$

The theoretically expected density matrix $\rho_{\text {the }}$ was obtained by applying the intended unitary transformation $U_{\text {the }}$ to the initial pseudo-pure state $\rho_{\text {ini }}$, as determined by state tomography. 


\section{RESULTS OF SPIN SQUEEZING EXPERIMENTS}

Using state tomography, ${ }^{(26)}$ the $2 j$ spin-1/2 density matrices $\rho_{\text {exp }}$ were reconstructed following squeezed state preparation for various values of the squeezing parameter $k=0, \ldots, \pi$. The expectation values and uncertainties along the basis axes were then calculated directly from these density matrices and the $J_{\mu}$ (or approximate $\tilde{J}_{\mu}$ matrices given in Eqs. (1) and (3)), as follows:

$$
\begin{aligned}
\left\langle J_{\mu}\right\rangle & =\operatorname{tr}\left(J_{\mu} \rho_{\text {exp }}\right), \\
\left\langle\Delta \mathbf{J}_{\mu}\right\rangle & =\sqrt{\operatorname{tr}\left(J_{\mu}^{2} \rho_{\exp }\right)-\left(\operatorname{tr}\left(J_{\mu} \rho_{\text {exp }}\right)\right)^{2}} .
\end{aligned}
$$

where $\mu=x, y, z$.

The correlation for the spin-1 pseudo-pure density matrix (see Fig. 4(i)) was 0.99 . For the density matrix corresponding to the maximally squeezed $(k=\pi / 2)$ spin-1 state, the correlation and the attenuated correlation were 0.99 and 0.98 , respectively. The correlation for the spin-3/2 pseudo-pure state density matrix (see Fig. 5(i)) was 0.98, while the correlation and the attenuated correlation of the maximally squeezed spin-3/2 state were 0.84 and 0.80 , respectively.

Since the squeezing operator conserves the total angular momentum, the combined $2 j$ spin- $1 / 2$ system should stay in the spin- $j$ subspace during the course of the experiment. However, due to decoherence and other errors in the implementation there is some "leakage" out of the effective spin- $j$ subspace. To quantify the accuracy with which we have been able to simulate the spin- $j$ system, we computed the best pseudo-pure-state approximation to mixed-state density matrix $\rho_{\text {exp }}$ by taking the eigenvector $\left|\psi_{\max }\right\rangle$ associated with the largest eigenvalue of $\rho_{\text {exp. }}$. The probability of leakage was then obtained from the definition

$$
\operatorname{Pr}_{\text {leak }}=1-\sum_{m=-j}^{j}\left|\left\langle j, m \mid \psi_{\max }\right\rangle\right|^{2}
$$

where $|j, m\rangle$ are the basis states of spin- $j$ subspace. This probability of leakage, averaged over all the experiments performed, was (0.02 \pm $0.02) \%$ for the spin-1 simulations and $(7.67 \pm 3.20) \%$ for the spin-3/2. The substantially larger leakage in the latter case was due to the fact that the protons were not decoupled from the carbons during the carbon scalar coupling evolution delays, in order to avoid the possibility of carbon-proton nuclear Overhauser effects. 


\subsection{The Spin-1 Case}

The initial pseudo-pure state corresponds to a coherent state of the embedded spin-1 subspace. It remains in a coherent spin state after a $\pi / 2$ rotation to the $x$-axis and accordingly, state tomography at this point reveals that $\left\langle\mathbf{J}_{x}\right\rangle$ is nearly equal to 1 while the spin uncertainties in the $y$ and $z$-directions are nearly equal to $1 / \sqrt{2}$. After applying the non-linear interaction for a period of $2 f(k)=k /(\pi J)$, the spin-1 expectation values in all directions are all close to 0 , consistent with maximally entangled state (see Table 3). The uncertainty is now maximum in the $x$-direction, while the uncertainties in the $y$ and $z$-directions are still nearly equal to $1 / \sqrt{2}$ because the principle axes of the squeezed ellipsoid are at $\pi / 4$ to the $y$ and $z$ axes (see Fig. 1(iii)). To make the squeezing more readily apparent, it was convenient to follow the squeezing step by a $\pi / 4 x$-pulse so that the uncertainty along the $z$-axis becomes 1 . The experimental and theoretical data for this maximally squeezed and rotated state are given in Table 3.

Table 3. Theoretical and Experimental Spin Expectation Values and Uncertainties of the Maximally Squeezed Spin-1 States

\begin{tabular}{lrrrrrr}
\hline & $\left\langle\mathbf{J}_{x}\right\rangle$ & $\left\langle\mathbf{J}_{y}\right\rangle$ & $\left\langle\mathbf{J}_{z}\right\rangle$ & $\left\langle\Delta \mathbf{J}_{x}\right\rangle$ & $\left\langle\Delta \mathbf{J}_{y}\right\rangle$ & $\left\langle\Delta \mathbf{J}_{z}\right\rangle$ \\
\hline Theory & 0.00 & 0.00 & 0.00 & 1.00 & 0.00 & 1.00 \\
Experiment & 0.00 & -0.02 & 0.00 & 0.97 & 0.21 & 0.98 \\
\hline
\end{tabular}

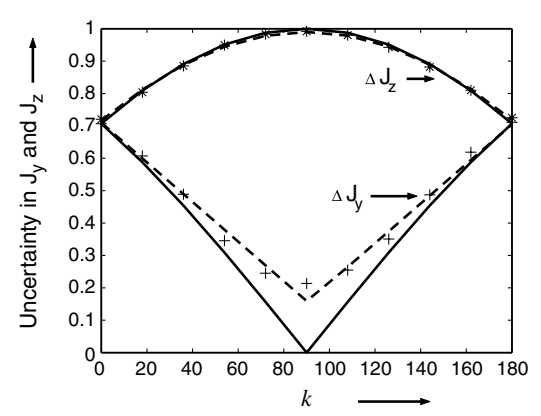

(i)

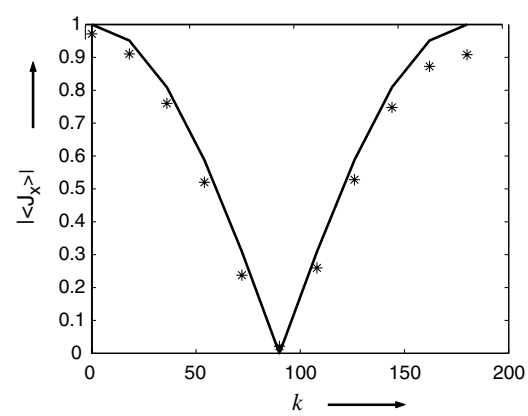

(ii)

Fig. 6. Plots of the theoretically expected uncertainties as a function of the squeezing parameter $k$ (in degrees) for spin-1 (solid lines), along with the fits (dashed lines) to the uncertainties computed from the experimental density matrices (+ or $*$ ). (a) Plots of $\left\langle\Delta \mathbf{J}_{y}\right\rangle$ (lower curves), and $\left\langle\Delta \mathbf{J}_{z}\right\rangle$ with k (upper curves). (b) Plot of $\left|\left\langle\mathbf{J}_{x}\right\rangle\right|$ (no fits shown). 
The variation of $\left|\left\langle J_{x}\right\rangle\right|, \Delta J_{y}$ and $\Delta J_{z}$ for different values of $k$ is plotted in Fig. 6. The corresponding values of $\left|\left\langle J_{y}\right\rangle\right|$ and $\left|\left\langle J_{z}\right\rangle\right|$ are close to zero implying that the spin system is polarized along the $x$ direction for all values of the squeezing parameter $k$.

\subsection{The Spin-3/2 Case}

After the initial $\pi / 2$ rotation to the $x$-axis, state tomography showed that the spin-3/2 angular momentum vector pointed in the $x$-direction with $\left\langle\mathbf{J}_{x}\right\rangle \approx 1.5$, and uncertainties in the $y$ and $z$-directions nearly equal to their theoretical values of $\sqrt{3} / 2$. Application of the non-linear interaction for a given $k$ created some apparent entanglement in the system, as indicated by the fact that $\left\langle\mathbf{J}_{x}\right\rangle$ was reduced to about 1 while $\left\langle\mathbf{J}_{y}\right\rangle$ and $\left\langle\mathbf{J}_{z}\right\rangle$ remained zero. The uncertainty in the $x$-direction also increased from 0 to $\sqrt{3} / 2$. To orient the axes of the squeezed uncertainty ellipsoid along the $y$ and $z$-directions, the results of state tomography were rotated about the $x$ axis (on a computer, since unlike the spin-1 case the rotation angle needed depends on the value of $k$ ). ${ }^{(14)}$ The experimental and theoretical data for the maximally squeezed state are shown in Table 4, while the variation of $\left|\left\langle J_{x}\right\rangle\right|, \Delta J_{y}$ and $\Delta J_{z}$ for different values of $k$ is plotted in Fig. 7. As in the spin-1 case, the corresponding values of $\left|\left\langle J_{y}\right\rangle\right|$ and $\left|\left\langle J_{z}\right\rangle\right|$ are close to zero, implying that the spin system is polarized along $x$-direction for all values of the squeezing parameter $k$, as desired.

\subsection{Behavior of Entanglement Measures}

The probability of leakage measurements described above (see Eq. (10)) show that the best pseudo-pure-state approximation to the final mixed-state density matrix quite accurately describes the spin- $j$ system. Thus this pseudo-pure state may be used to study the entanglement of the constituent spin-1/2 particles by well-understood pure-state entanglement criteria. The criteria used here are the entanglement of formation (or, for

Table 4. Theoretical and Experimental Spin Expectation Values and Uncertainties of the Maximally Squeezed Spin-3/2 States

\begin{tabular}{lcccccc}
\hline & $\left\langle\mathbf{J}_{x}\right\rangle$ & $\left\langle\mathbf{J}_{y}\right\rangle$ & $\left\langle\mathbf{J}_{z}\right\rangle$ & $\left\langle\Delta \mathbf{J}_{x}\right\rangle$ & $\left\langle\Delta \mathbf{J}_{y}\right\rangle$ & $\left\langle\Delta \mathbf{J}_{z}\right\rangle$ \\
\hline Theory & 1.00 & 0.00 & 0.00 & 0.87 & 0.50 & 1.32 \\
Experiment & 0.82 & 0.05 & -0.13 & 0.92 & 0.53 & 1.36 \\
\hline
\end{tabular}




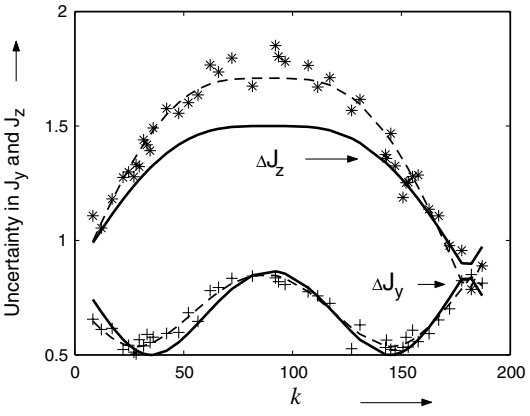

(i)

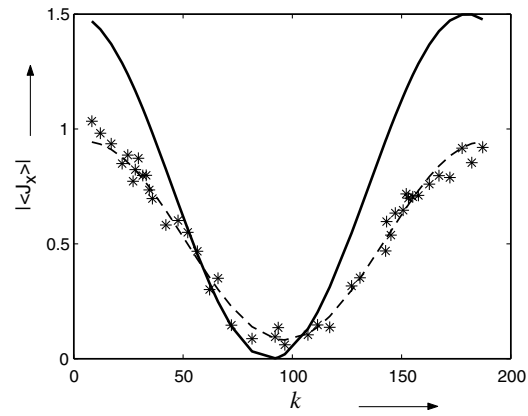

(ii)

Fig. 7. Plots of the theoretically expected uncertainties as a function of the squeezing parameter $k$ (in degrees) for spin-3/2 (solid lines), along with fits (dashed lines) to the uncertainties computed from the experimental density matrices ( + or $*$ ). (i) Plots of $\left\langle\Delta \mathbf{J}_{y}\right\rangle$ (lower curves) and $\left\langle\Delta \mathbf{J}_{z}\right\rangle$ (upper curves). (ii) Plot of $\left|\left\langle\mathbf{J}_{x}\right\rangle\right|$.

bipartite pure states, the von Neumann entropy of the partial trace over either subsystem), ${ }^{(28)}$ and the concurrence ${ }^{(29,30)}$ of (the partial trace onto any) pair of qubits. The purpose of this discussion is not to uncover any new features of entanglement in these simple systems, but rather to use the compatibility of the experimental results with the well-known behavior of these entanglement measures as a benchmark for the precision of control obtained. In addition, these entanglement measures were computed without taking into account the very large identity component that is always present in liquid-state NMR, and hence should be regarded as measures of the "pseudo-entanglement" associated with the pseudo-pure states used for the experiments.

Figure 8 shows plots of the theoretically expected and experimentally observed entanglement measures as a function of the squeezing parameter $k$ for the spin- 1 experiments. It is immediately apparent that the theory and experiments agree extremely well with respect to either entanglement measure, in accord with the fact that the concurrence and entanglement of formation are monotonically related for two qubits. The maximally squeezed state $\left(k=90^{\circ}\right)$ is observed to correspond to the maximally entangled state, as theory predicts it should in a representation by Dicke states. $^{(31)}$

Figure 9 shows the analogous pair of plots for the spin-3/2 experiments, where the interpolation is now done using a fifth-order polynomial fit to the data (dashed lines). In this case three different entanglements of formation are obtained, depending on which pair of qubits is traced over in order to obtain the reduced density matrix, and 


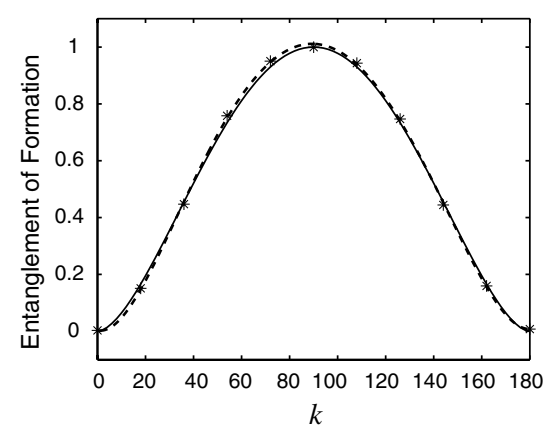

(i)

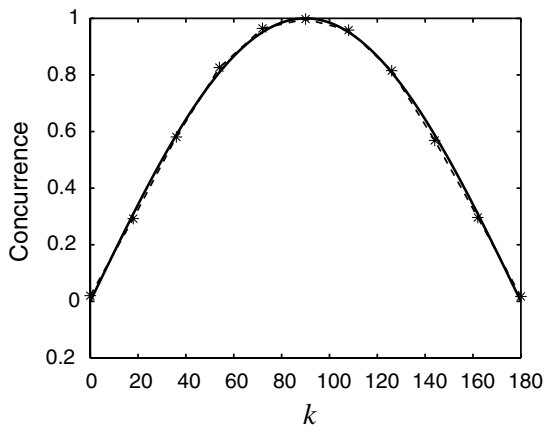

(ii)

Fig. 8. Plots of the entanglement of formation (i) and concurrence (ii) with the squeezing parameter $k$ (in degrees) for spin-1. The solid lines are the theoretically expected curves, while the dashed lines interpolate linearly between the values computed from the experimental density matrices obtained via tomography $(*)$.

their average, also known as the Meyer's entanglement metric, ${ }^{(32)}$ is plotted for simplicity. According to this metric, the maximum entanglement again occurs at $k=90^{\circ}$ and corresponds to a Greenberger-Horne-Zeilinger state, which is however not a maximally squeezed state. The maximally squeezed state now occurs instead at $k=34.7^{\circ}$ and again at 180-34.7 = $155.3^{\circ}$, and corresponds to the maximum concurrence of the reduced density matrix obtained by tracing over any one qubit (which is attained by a $W$-state). The correspondence between theory and experiment in this case is noticeably lower than in the spin-1 experiments, primarily because of leakage from the carbons used as qubits into the alpha and methyl protons during the experiment.

\section{CONCLUSIONS}

We have demonstrated the use of liquid-state NMR to simulate squeezed states of the effective spin- 1 and $3 / 2$ subsystem contained in a two and three-qubit system, respectively. We have further shown that the precision of quantum control obtained was sufficient to reproduce the theoretically expected behavior of the spin- 1 and $3 / 2$ observables as well as the associated entanglement measures among the underlying qubits. The results are a further demonstration of the utility of pseudo-pure states, ${ }^{(22)}$ and the power of strongly modulating pulses, ${ }^{(19)}$ for the development and validation of quantum control methods. The dynamics of the coherences 


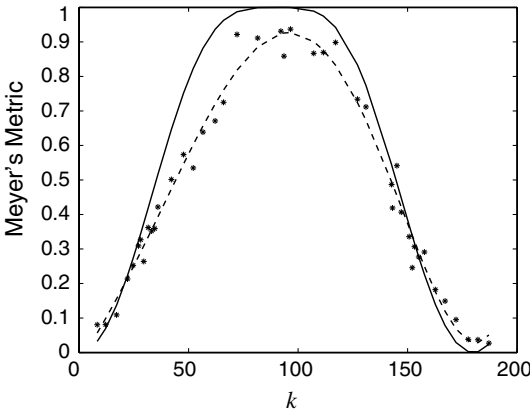

(i)

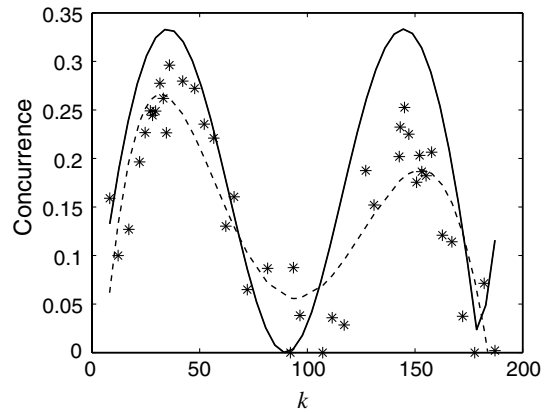

(ii)

Fig. 9. Plots of the Meyer's metric or average entanglement over tracing down to each of the three qubits in turn (i) and concurrence after tracing down to any pair of qubits (ii) with the squeezing parameter $k$ (in degrees) for spin-3/2. The solid lines are the theoretically expected curves, while the dashed lines are a fifth-order polyomial fit to the values computed from the experimental density matrices obtained via tomography $(*)$.

among the fiducial states that were confirmed by complete tomography are fully in accord with these proposals, even though the highly mixed states used in our experiments were, of course, separable at all times. It is interesting to observe, however, that even when the identity component of the density matrix is fully taken into account the uncertainties in the $x$ and $y$ directions were unequal, i.e., the actual mixed states created could be regarded as (very slightly) "squeezed".

It should also be pointed out that the interpretation of the higher spin states as "squeezed" or not depends on how the higher spin states are mapped into the symmetric subspace of the multi-spin- $1 / 2$ system. We discussed earlier, for example, how the mapping used here for the spin$3 / 2$ experiments actually gave the sum of a spin- $3 / 2$ with a spin- $1 / 2$ pair, limiting the degree of squeezing attainable. Even when the mathematical representation is strictly correct, however, the physical properties of the squeezed states can be rather different. For instance, if we were to replace the Dicke states $|\uparrow \uparrow\rangle$ and $|\downarrow \downarrow\rangle$ in Table 1 by the Bell states $1 / \sqrt{2}(|\uparrow \uparrow\rangle \pm$ $|\downarrow \downarrow\rangle)$, the maximally squeezed state of the simulated spin-1 would correspond to the unentangled basis state $|00\rangle$ of the two-qubit system in which it is contained. Such a representation may be a bit unnatural, since rotations of the qubits no longer correspond to rotations of the higher spin, but should still be kept in mind when discussing the relations between entanglement in multi-qubit systems and the squeezing of the effective higher spins therein. ${ }^{(4,16)}$ 


\section{ACKNOWLEDGMENTS}

We thank C. Ramanathan and T. Mahesh for assistance. This work was supported by ARO grant DAAD19-01-1-0519, the DARPA QuIST Program, and the Cambridge-MIT Institute.

\section{REFERENCES}

1. S. H. Chen and M. Kotlarchyk, Interactions of Photons and Neutrons with Matter (World Scientific, Singapore, 1997).

2. M. O. Scully, Quantum Optics (Cambridge University Press, Cambridge, 1997).

3. L.-M. Duan, A. Sorensen, J. I. Chirac, and P. Zoller, Phys. Rev. Lett. 85, 3991-3994 (2000).

4. M. Kitagawa and M. Ueda, Phys. Rev. A 47, 5138-5143 (1993).

5. A. Sorensen, L.-M. Duan, J. I. Cirac, and P. Zoller, Nature 409, 63-65 (2001).

6. D. J. Wineland, J. J. Bollinger, W. M. Itano, and F. L. Moore, Phys. Rev. A 46, 67976800 (1992).

7. D. J. Wineland, J. J. Bollinger, W. M. Itano, and D. J. Heinzen, Phys. Rev. A 50, 67-88 (1994).

8. K. Wodkiewicz, J. Mod. Opt. 34, 941 (1987).

9. K. Wodkiewicz and J. Eberly, J. Opt. Soc. Am. B 2, 458 (1985).

10. G. S. Agarwal and R. R. Puri, Phys. Rev. A 41, 3782-3791 (1990).

11. A. Sorensen and K. Molmer, Phys. Rev. A 83, 2274-2277 (1999).

12. A. Sorensen and K. Molmer, Phys. Rev. Lett. 83, 2274-2277 (1999).

13. A. Kuzmich, L. Mandel, and N. P. Bigelow, Phys. Rev. Lett., 85, 1594 (2000).

14. A. R. Edmonds, Angular Momentum in Quantum Mechanics (Princeton University Press, Princeton, NJ, 1974).

15. M. E. Rose, Elementary theory of Angular Momentum (Wiley, 1957, reprinted by Dover, Newyork, 1995).

16. A. R. Usha Devi, X. Wang, and B. C. Sanders, Quantum Inf. Process. 2, 207-220, (2003).

17. A. J. Shaka and R. Freeman, J. Magn. Reson. 55, 487-493 (1983).

18. E. M. Fortunato, M. A. Pravia, N. Boulant, G. Teklemariam, T. F. Havel, and D. G. Cory, J. Chem. Phys. 116, 7599-7606 (2002).

19. M. A. Pravia, N. Boulant, J. Emerson, A. Farid, E. M. Fortunato, T. F. Havel, R. Martinez, and D. G. Cory. J. Chem. Phys. 119, 9993-10001 (2003).

20. R. Freeman, Spin Choreography (Oxford University Press, oxford, 1998).

21. Y. S. Weinstein, M. A. Pravia, E. M. Fortunato, S. Lloyd, and D. G. Cory, Phys. Rev. Lett. 86, 1889-1891 (2001).

22. T. F. Havel, S. S. Somaroo, C.-H. Tseng, and D. G. Cory, in Proceedings of the Applicable Algebra in Engineering, Communications and Computing, vol. 10, Springer-Verlag, Berlin, 2000,pp. 339-374

23. R. Laflamme, E. Knill, D. G. Cory, E. M. Fortunato, T. F. Havel, C. Miquel, R. Martinez, C. Negrevergne, G. Ortiz, M. A. Pravia, Y. Sharf, S. Sinha, R. Somma, and L. Viola, Los Alamos Sci. 27, 2-37 (2002).

24. M. D. Price, T. F. Havel, and D. G. Cory, Phys. D 120, 82-101 (1998).

25. G. Teklemariam, E. M. Fortunato, M. A. Pravia, T. F. Havel, and D. G. Cory, Phys. Rev. Lett. 86, 5845-5849 (2001). 
26. M. A. Nielsen and I. L. Chuang, Quantum Computation and Quantum Information (Cambridge University Press, Cambridge, 2000).

27. R. Laflamme, E. Knill, W. H. Zurek, P. Catasti, and S. Marathan, Phil. Trans. R. Soc. Lond. A 356, 1941-1948 (1998).

28. C. H. Bennett, D. P. DiVincenzo, J. A. Smolin, and W. K. Wootters, Phys. Rev. A 54, 3824 (1996).

29. S. Hill and W. K. Wootters, Phys. Rev. Lett. 78, 5022-5025 (1997).

30. W. K. Wootters, Phys. Rev. Lett. 80, 2245-2248 (1998).

31. Xiaoguang Wang and K. Molmer, Eur. Phys. J. D 18, 385-391 (2002).

32. D. A. Meyer and N. R. Wallach, J. Math. Phys. 43, 4273-4278 (2002). 